\title{
Multi-wavelength environment of the Galactic globular cluster Terzan 5
}

\author{
A.-C. Clapson ${ }^{1,2}$, W. Domainko ${ }^{1}$, M. Jamrozy ${ }^{3}$, M. Dyrda ${ }^{4}$, and P. Eger $^{5}$ \\ ${ }^{1}$ Max-Planck-Institut für Kernphysik, PO Box 103980, 69029 Heidelberg, Germany \\ e-mail: clapson@mpi-hd.mpg.de \\ 2 EMBL Heidelberg, Meyerhofstrasse 1, 69117 Heidelberg, Germany \\ 3 Obserwatorium Astronomiczne, Uniwersytet Jagielloński, ul. Orla 171, 30-244 Kraków, Poland \\ ${ }^{4}$ Instytut Fizyki Jạdrowej PAN, ul. Radzikowskiego 152, 31-342 Kraków, Poland \\ 5 Universität Erlangen-Nürnberg, Physikalisches Institut, Erwin-Rommel-Str. 1, 91058 Erlangen, Germany
}

Received 10 August 2010 / Accepted 12 May 2011

\begin{abstract}
Terzan 5 is a Galactic globular cluster exhibiting prominent X-ray and gamma-ray emission. Following the discovery of extended $\mathrm{X}$-ray emission in this object, we explore here archival data at several wavelengths for other unexpected emission features in the vicinity of this globular cluster. Radio data from the Effelsberg 100-m telescope show several extended structures near Terzan 5, albeit with large uncertainties in the flux estimates and no reliable radio spectral index. In particular, a radio source extending from the location of Terzan 5 to the north-west could result from long-term non-thermal electron production by the large population of milli-second pulsars in this globular cluster. Another prominent radio structure close to Terzan 5 may be explained by ionised material produced by a field $\mathrm{O}$ star. As for the diffuse X-ray emission found in Terzan 5, its extension appears to be limited to within 2.5 arcmin of the globular cluster and the available multi-wavelength data is compatible with an inverse Compton scenario but disfavours a nonthermal Bremsstrahlung origin.
\end{abstract}

Key words. globular clusters: individual: Terzan 5 - radiation mechanisms: general - stars: massive

\section{Introduction}

Terzan 5 is a Galactic globular cluster (GC) located in the inner Galaxy, at RA $17^{\mathrm{h}} 48^{\mathrm{m}} 04.0^{\mathrm{s}}$ and Dec $-24^{\circ} 46^{\prime} 45^{\prime \prime}$ (in Galactic coordinates: $l 3.8^{\circ}, b 1.69^{\circ}$ ), at $5.9 \mathrm{kpc}$ from the Sun (Ferraro et al. 2009). Terzan 5 hosts the largest population of millisecond pulsars (msPSRs) detected so far (33, Ransom 2008), and it exhibits the highest central stellar density among Galactic GCs (Lanzoni et al. 2010).

Recent observational results on Terzan 5 concerning nonthermal signatures have renewed the interest in this object. Eger et al. (2010) report diffuse X-ray emission extending beyond the half-mass radius of the $\mathrm{GC}\left(r_{\mathrm{h}}=0.52^{\prime}\right.$, Lanzoni et al. 2010) with a hard spectrum of index close to 1 , likely of non-thermal origin. In the high-energy $\gamma$-ray range $\left(100 \mathrm{MeV}<E_{\gamma}<100 \mathrm{GeV}\right)$ Terzan 5 is the brightest GC seen with Fermi-LAT (Kong et al. 2010; Abdo et al. 2010). The $\gamma$-ray spectrum of the source is best fitted by a power law with an exponential cut-off at a few $\mathrm{GeV}$, as expected for a population of msPSRs. Kong et al. (2010) also report tentative evidence of a second component in the $\gamma$-ray spectrum at energies above $10 \mathrm{GeV}$, which they interpret as inverse Compton (IC) up-scattering of cluster stellar photons by highenergy electrons. These observations clearly point to a considerable population of non-thermal particles in Terzan 5 .

Several models have been proposed that could explain the observed high-energy emission, based for some on leptons accelerated by the population of msPSRs in the GC (e.g. see Bednarek \& Sitarek 2007; Venter \& de Jager 2008; Venter et al. 2009). These models apply in principle to any GC hosting msPSRs. The largest predicted fluxes (based on the known msPSRs) are from 47 Tucanae, also detected by Fermi-LAT (Abdo et al. 2009) and Terzan 5, where Abdo et al. (2010) estimate a population of 180 msPSRs, with a large uncertainty. Since different emission processes may dominate in the $\mathrm{GeV}$ range and at much lower energies, there is no reason a priori for 47 Tucanae to be brighter than Terzan 5 in all parts of the spectrum. Another contribution to the high-energy emission may come from stellar binaries consisting of a normal star and a compact object, accelerating particles to the required energies possibly via non-thermal radio-emitting plasma ejections (described e.g. in Fender et al. 1999). Due to its high core density favourable to dynamical interactions between stellar objects, Terzan 5 is expected to contain many binary systems (e.g. Pooley \& Hut 2006; Ivanova et al. 2008). This would also favour merger events, including white dwarf mergers (see e.g. Shara \& Hurley 2002) and neutron star mergers (discussed in Grindlay et al. 2006), expected to result in explosive events, type Ia supernovae or short gamma-ray bursts (GRBs) depending on the progenitors (white dwarfs and neutron stars respectively). The remnants of these explosions may then be particle-acceleration sites (see e.g. Koyama et al. 1995; Domainko \& Ruffert 2005, 2008).

A scenario for extended X-ray emission in relation to GCs is proposed in Okada et al. (2007). They suggest that supersonic motion through the interstellar medium (ISM) and bowshock formation are possible for GCs. They also find potential signatures of particles accelerated in GC bow-shocks in radio and X-ray data. Typical three-dimensional velocities of GC are around $200 \mathrm{~km} \mathrm{~s}^{-1}$, but can be as high as $400 \mathrm{~km} \mathrm{~s}^{-1}$ for, e.g., 
NGC 5904 (Okada et al. 2007). Proper motion is unfortunately not available for Terzan 5. Its radial velocity, a lower limit to the total three-dimensional velocity, is about $80 \mathrm{~km} \mathrm{~s}^{-1}$ (Harris 1996).

Previous radio studies of Galactic GCs (see e.g. Gopal-Krishna \& Steppe 1980) have reported flat-spectrum radio sources, some of which (M 3 and M 92) surrounded by arcmin-scale radio features.

Many studies have focused on the GC Terzan 5 itself, if not on its core. Our aim here, prompted by the discovery of X-ray diffuse emission (Eger et al. 2010), is to collect information from archival data over spatial scales from the arcmin to the degree, with particular interest in indications of non-thermal emission processes. This paper is organised as follows. First, measurements from archival data of the region around Terzan 5 are presented. Radio data are discussed in Sect. 2, X-ray in Sect. 3, and infrared in Sect. 4.1. The density of molecular material is investigated in Sect. 4.2 and possible counterparts from astronomical catalogues are mentioned in Sect. 5. Then, in Sect. 6 the implication of our findings on the underlying physical processes are discussed.

\section{Radio survey data}

Fruchter \& Goss (2000) observed Terzan 5 at wavelengths of 90, 20 and $6 \mathrm{~cm}$ with high angular-resolutions using the VLA in its three most extended configurations. Their radio maps display numerous point sources with steep spectra, as expected for pulsars. Terzan 5 is also detected in the $21 \mathrm{~cm}$ NVSS survey (Condon et al. 1998) as a 5 mJy single source, with a weak polarisation feature extending north of the GC.

\subsection{Effelsberg survey data}

For the radio analysis of Terzan 5 we used archival 21 and $11 \mathrm{~cm}$ (respectively 1408 and $2695 \mathrm{MHz}$ ) total intensity maps from the Survey Sampler ${ }^{1}$ of the Max Planck Institute for Radio Astronomy. These observations were done with the Effelsberg $100-\mathrm{m}$ telescope as part of Galactic plane surveys. Since we are interested in small-scale structures, we used the "sourcecomponent" maps, which do not contain the global steepspectrum diffuse Galactic emission. Full details on the data and the processing can be found in Reich et al. (1990a,b). The analysis presented in this paper relies on this preprocessing. The flux density (S) calibration for the dataset was checked by mapping the point-like source 3C286 and adopting the scale of Baars et al. (1977): flux densities of 14.4 (at $21 \mathrm{~cm}$ ) and $10.4 \mathrm{Jy}$ (at $11 \mathrm{~cm}$ ). The angular resolution (half-power beam width, $H P B W$ ) of the maps are 9.'4 and 4.'3, their sensitivity (noise rms) 40 and $20 \mathrm{mK} T_{\mathrm{B}}$, and the ratios $T_{\mathrm{B}}[\mathrm{K}] / \mathrm{S}[\mathrm{Jy}]$ are $2.03 \pm 0.04$ and $2.51 \pm 0.05$, respectively for 21 and $11 \mathrm{~cm}\left(T_{\mathrm{B}}\right.$ the brightness temperature). The resulting flux maps are shown in Figs. 1 and 4.

\subsection{Regions of interest}

The regions used in this work were defined from visual inspection of the Effelsberg radio data. Several features can be seen around Terzan 5, but none exhibits tell-tale morphology (e.g. shell-like or bow-shock-like structure) suggestive of its origin. Ring segments centred on the GC were defined to cover its environment. The width of the rings matches the angular resolution of the radio data, while their orientation aims at isolating main

\footnotetext{
${ }^{1}$ http://www .mpifr-bonn.mpg.de/survey.html
}

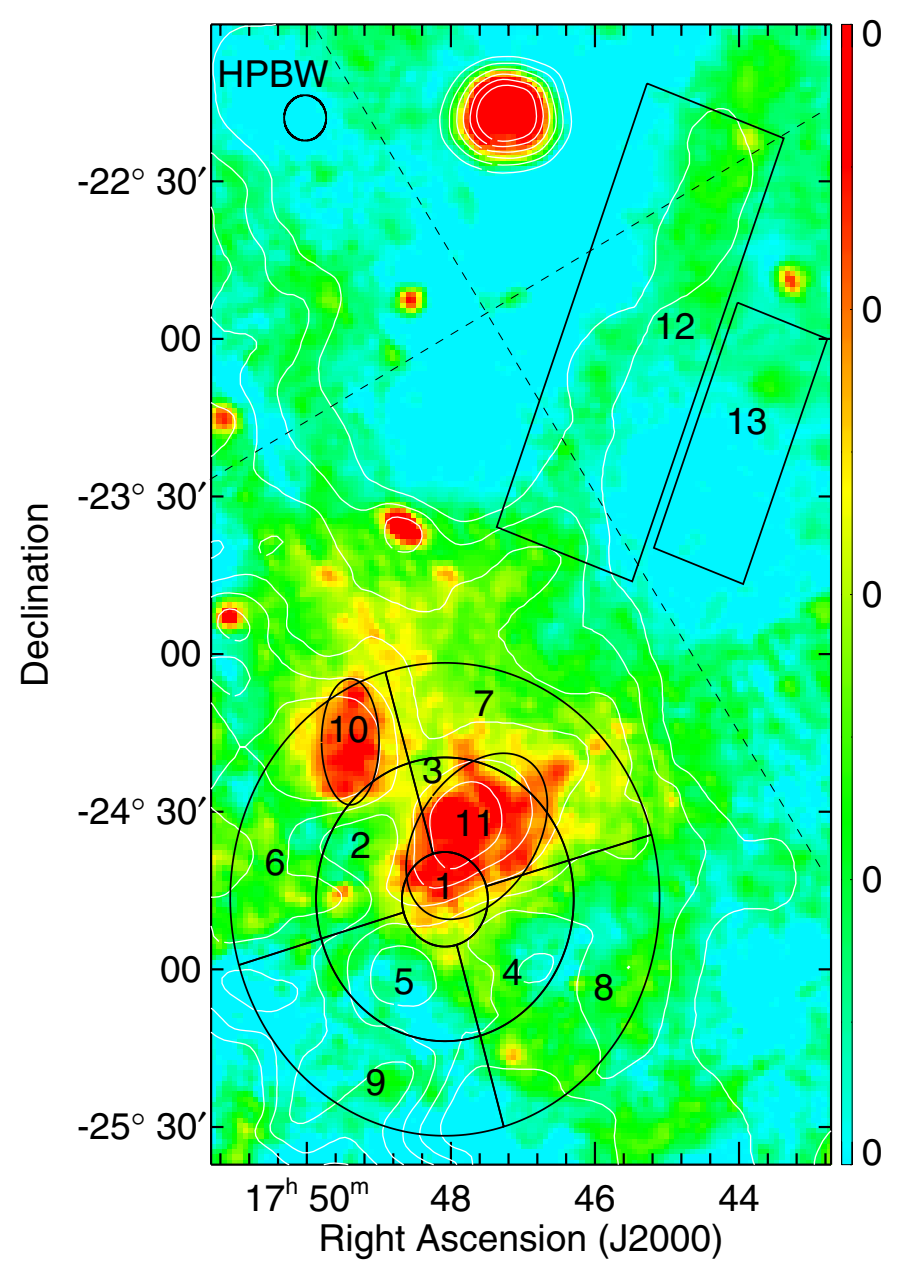

Fig. 1. Radio map from the Effelsberg Galactic plane surveys at $11 \mathrm{~cm}$ (in Jy/beam). Contours from the $21 \mathrm{~cm}$ map are over-plotted (for levels 0.2 to $0.7 \mathrm{Jy} /$ beam per $0.1 \mathrm{step}$ ), as well as the regions of Table 1 . The region labelled 1 is centred on Terzan 5. The $H P B W$ for the $11 \mathrm{~cm}$ data is also shown. The dashed lines indicate the radio scan directions at $11 \mathrm{~cm}$.

flux levels differences. Two elliptical regions and a rectangular region cover distinct prominent higher flux features, visible in Fig. 1. The geometry of all the regions is given in Table 1 and shown in Fig. 1.

The radio flux densities of the regions were extracted using the AIPS $^{2}$ task IRING, or IMEAN for regions 12 and 13. The error on the radio flux in a region was derived following Klein et al. (2003):

$\Delta S_{i}=\sqrt{\left(S_{i} \times \Delta S_{\mathrm{c}}\right)^{2}+\left(\Delta S_{i}^{n}\right)^{2}+\left(\Delta S_{i}^{z}\right)^{2}}$

for $S_{i}$ the flux measurement at frequency $v_{\mathrm{i}}$ and $\Delta S_{i}$ the related error. We used a calibration error $\Delta S_{\mathrm{c}}$ of $3 \%$ and a noise rms $\Delta S_{i}^{n}$ derived from the rms noise of the map (respectively 0.08 and $0.05 \mathrm{Jy} /$ beam at 21 and $11 \mathrm{~cm}$ ) and multiplied the results by the integration area. The estimation of a "diffuse-component" performed by Reich et al. (1990a,b) may locally produce large inaccuracies. The "diffuse-component" was subtracted from the original data to obtain the "source-component" map used here. We consider a zero-level error $\Delta S_{i}^{z}$ at $3 \%$ of the mean flux value in the region of the "diffuse-component" map. $\Delta S_{\mathrm{i}}^{z}$ also depends

${ }^{2}$ http://www.aips.nrao.edu/ 
Table 1. Description of the regions defined from the radio maps.

\begin{tabular}{lccccc}
\hline \hline $\begin{array}{l}\text { Region } \\
\text { number }\end{array}$ & $R_{\min }$ & $\begin{array}{c}R_{\max } \\
{[\mathrm{deg}]}\end{array}$ & $\begin{array}{c}\phi_{\operatorname{mix}} \\
\text { Area } \\
{\left[10^{-6} \mathrm{sr}\right]}\end{array}$ \\
\hline 1 & 0.0 & 0.15 & 0 & 360 & 22.59 \\
2 & & & -106 & -16 & \\
3 & 0.15 & 0.45 & -16 & 74 & 45.17 \\
4 & & & 74 & 164 & \\
5 & & & 164 & -106 & \\
6 & & & -106 & -16 & \\
7 & 0.45 & 0.75 & -16 & 74 & 90.34 \\
8 & & & 74 & 164 & \\
9 & & & 164 & -106 & \\
10 & 0.1 & 0.2 & 0 & - & 19.13 \\
11 & 0.2 & 0.3 & 40 & - & 57.39 \\
12 & 0.5 & 1.5 & 20 & - & 228.46 \\
13 & 0.33 & 0.83 & 20 & - & 84.61 \\
\hline
\end{tabular}

Notes. Region 1 is a circle, regions 2 to 9 are circle wedges, all centred on the core position of Terzan 5. Regions 10 and 11 are ellipses, of centres RADec $\left(267.35^{\circ},-24.28^{\circ}\right)$ and $\left(266.91^{\circ},-24.58^{\circ}\right)$, major and minor axes $R_{\max }$ and $R_{\min }$ and inclination $\phi_{\min }$. Region 12 is a rectangular box, centred on RADec $\left(266.35^{\circ},-22.98^{\circ}\right)$, of side lengths $R_{\min }$ and $R_{\max }$ and inclination $\phi_{\min }$. The rotation angles are relative to north, westwards.

on the area (in units of solid angle, used to determine the zerolevel). $\Delta S_{i}^{z}$ is the predominant contribution to the flux errors.

The flux density values are summarised in Table 2 . Regions 4, 5 and 8 might indicate local background levels at 11 and $21 \mathrm{~cm}$, with a possible deviation for region 9 (below at $11 \mathrm{~cm}$, above at $21 \mathrm{~cm}$ ). While two bright regions (10 an 11) stand out at both wavelengths, it is difficult to make out other features, in particular at $21 \mathrm{~cm}$, where the errors are larger and the resolution worse. Regions 1, 2, 3, 6 and 7 overlap somewhat with the bright features and present as expected intermediate flux values.

Region 12 is visible only because of the very low flux densities in its surroundings. Since it is located at higher galactic latitudes the background level of the radio emission is considerably lower than close to Terzan 5. To quantify the robustness of the structure we estimated the background level of the flux density in region 13, adjacent to region 12 at the same galactic latitude (see Table 2). At least at $21 \mathrm{~cm}$, region 12 stands significantly above the local background level.

\subsection{Spectral index estimation}

We extracted the mean spectral index $\alpha$ for each region from the two flux measurements in each region. The uncertainty on $\alpha$, estimated following Eq. (3) of O'Dea et al. (2009) is very large in all regions. We therefore do not present the radio spectral information in this paper. For the discussion we use a spectral index of -0.8 (see Sect. 6). Detailed re-analysis of the archival data or additional data spanning a wider frequency range may reduce this limitation.

\section{X-ray data}

\subsection{ROSAT}

The first detection of extended diffuse emission from the GC Terzan 5 was reported by Eger et al. (2010) who analysed the data of an archival Chandra Advanced CCD Imaging Spectrometre (ACIS) observation. Following this discovery we investigated archival ROSAT data, to search for diffuse emission on a much larger spatial scale than possible with the limited field of view (FoV) of Chandra. Terzan 5 was observed by ROSAT several times (see e.g. Johnston et al. 1995). We focused on a pointing observation centred on Terzan 5 taken in 1991 (ID 300060) by the High Resolution Imager (HRI) instrument with a total live time of $22.5 \mathrm{ks}$. The advantage of the ROSAT-HRI observation compared to Chandra is its large square FoV with a side of $38^{\prime}$. Data analysis relied on the standard packages FTOOLS 5.9 from HEASOFT version 6.8 (Blackburn 1995) and CIAO ${ }^{3}$.

To search for extended diffuse emission, point-like sources must first be excluded. There are 50 Chandra point-sources inside $r_{\mathrm{h}}$, listed in Table 1 of Heinke et al. (2006). To account for the difference between the point spread function (PSF) of ROSAT-HRI ( $5^{\prime \prime}$, Boese 2000) and Chandra-ACIS $\left(\sim 1^{\prime \prime}\right.$, Karovska et al. 2001), we defined a large exclusion region at the centre of the GC, with a radius equal to $r_{\mathrm{h}}$. Outside $r_{\mathrm{h}}$, Heinke et al. (2006) found 78 Chandra sources (listed in their Table 2). We excluded them taking into account the HRI PSF. All the excluded regions were removed from the dataset and refilled with the CIAO DMFILTH tool, assuming Poisson statistics.

Since the spectral resolution of the HRI camera is very limited, we only extracted the total count-rate in the full HRI energy band (0.1-2.4 keV). The HRI background is discussed in the ROSAT-HRI calibration report (David et al. 1997). It combines different components: the internal background, the externallyinduced background from cosmic rays and the X-ray background (Galactic and extra-galactic). For our analysis we adopted the given standard values for the first two (pointing-independent) components. Terzan 5 is located near the Galactic plane, therefore the X-ray background, in particular the diffuse Galactic emission, is larger than average over the sky. Based on the results from Eger et al. (2010), we estimated this component as $3 \times 10^{-7}$ counts s $^{-1} \operatorname{arcsec}^{-2}$. Compared to David et al. (1997), this is slightly above the standard value but still within the given range. The total expected HRI background level for this observation amounts then to $1.2 \times 10^{-6}$ counts s$^{-1} \operatorname{arcsec}^{-2}$ in the 0.1-2.4 keV energy band.

Diffuse emission connected to Terzan 5 was searched for using concentric annular extraction regions $1^{\prime}$ thick centred on the $\mathrm{GC}$, of internal radii 0 to $10^{\prime}$. We integrated the counts within each ring from the refilled HRI image and compared these to the afore-mentioned background component level. Figure 2 shows the surface brightness for the rings. All the extracted values agree within $1 \sigma$ and are compatible with the expected background. The innermost region, somewhat brighter than the others, is still within less than $2 \sigma$ deviation from the expected background. The diffuse emission detected with Chandra (Eger et al. 2010) as well as un-removed point sources are likely to contribute to the flux measured in this innermost ring. In addition there might still be some contamination from the wings of the PSF of removed point-sources or a contribution from a transient source, a common phenomenon in dense GCs like Terzan 5 (see e.g. Heinke et al. 2002).

The archival HRI data confirm the previous results of Eger et al. (2010) and show no indication of emission above the Galactic diffuse component beyond 2'.5 away from Terzan 5. The extended X-ray emission connected to Terzan 5 seems therefore to be very localised, a result not accessible with the smallFoV Chandra data analysed in Eger et al. (2010).

\footnotetext{
${ }^{3}$ http://cxc.harvard.edu/ciao/
} 
Table 2. Measurements extracted for the regions from the Effelsberg radio maps (Reich et al. 1990a,b).

\begin{tabular}{|c|c|c|c|c|c|c|c|c|c|c|c|c|c|}
\hline \multirow[b]{3}{*}{$\begin{array}{l}\text { Wavelength } \\
{[\mathrm{cm}]}\end{array}$} & \multicolumn{13}{|c|}{ Region } \\
\hline & 1 & 2 & 3 & 4 & 5 & 6 & 7 & 8 & 9 & 10 & 11 & 12 & 13 \\
\hline & \multicolumn{13}{|c|}{$\begin{array}{l}\text { Average radio flux density (error) } \\
{[\mathrm{kJy} / \mathrm{sr}]}\end{array}$} \\
\hline \multirow[t]{2}{*}{11} & 62.60 & 39.56 & 64.44 & 28.16 & 18.31 & 41.39 & 45.06 & 25.56 & 8.41 & 61.58 & 67.21 & 12.89 & 7.00 \\
\hline & (9.39) & $(6.87)$ & (6.43) & $(6.35)$ & (6.94) & $(5.10)$ & $(4.42)$ & $(4.35)$ & $(5.21)$ & $(10.20)$ & $(5.85)$ & $(2.04)$ & $(6.94)$ \\
\hline \multirow[t]{2}{*}{21} & 60.88 & 63.76 & 74.05 & 33.94 & 40.69 & 81.75 & 55.71 & 35.54 & 50.63 & 86.67 & 73.53 & 18.54 & 1.29 \\
\hline & (17.74) & (12.73) & (11.84) & (11.93) & (12.91) & $(9.47)$ & $(8.00)$ & $(8.23)$ & (9.66) & (18.97) & (10.67) & $(4.07)$ & $(4.30)$ \\
\hline
\end{tabular}

Notes. Regions 4, 5, 8 and 13 might indicate local background levels at 11 and $21 \mathrm{~cm}$.

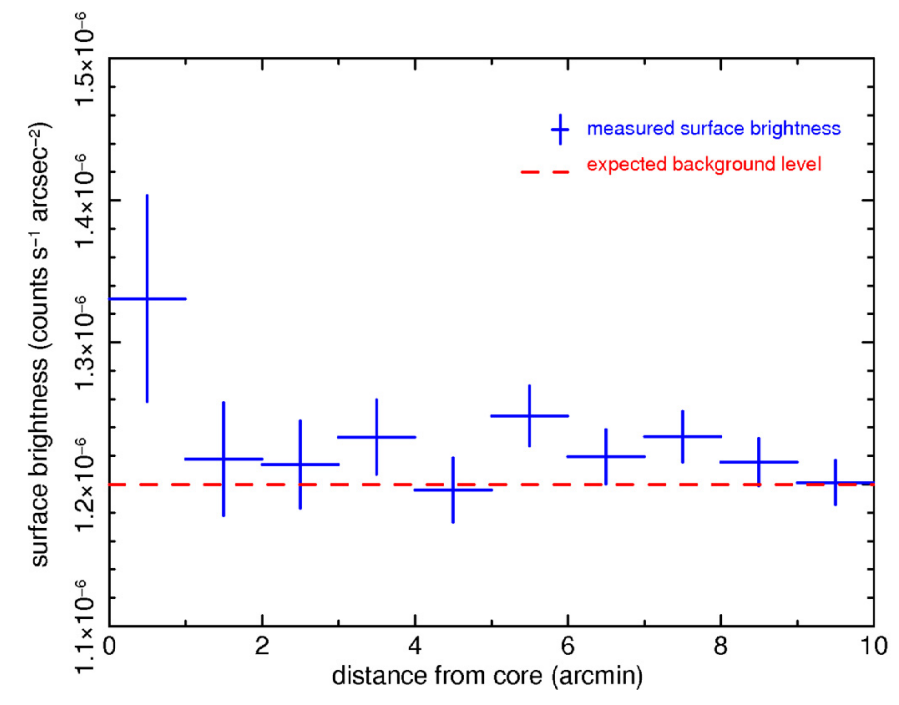

Fig. 2. ROSAT-HRI surface brightness versus distance from the core position of Terzan 5 , with a $1 \sigma$ error bar. The expected background level is represented by the dashed horizontal line. The extraction regions are adjacent rings of equal width, see text for details.

\subsection{INTEGRAL}

The non-thermal X-ray emission detected by Eger et al. (2010) might be detectable in the hard X-ray regime by INTEGRAL if it extends to this energy band. For this source, of unabsorbed flux $5.5 \times 10^{-13} \mathrm{erg} \mathrm{cm}^{-2} \mathrm{~s}^{-1}$ and spectral index of about 0.9 in the $1-7 \mathrm{keV}$ band (Eger et al. 2010), a flux of $4.9 \times 10^{-12} \mathrm{erg} \mathrm{cm}^{-2} \mathrm{~s}^{-1}$ in the $17-60 \mathrm{keV}$ band is expected. This might be within the reach of INTEGRAL, which can detect sources down to $3.7 \times 10^{-12} \mathrm{erg} \mathrm{cm}^{-2} \mathrm{~s}^{-1}$ in the same band (Krivonos et al. 2010) near the Galactic centre (and also at the position of Terzan 5).

\section{Other wavelengths}

Infrared and ${ }^{12} \mathrm{CO}$ survey data were also studied, to gather information about the environment from dust and molecular gas content. A gradient in infrared emission in the direction perpendicular to the Galactic plane was found. For the whole region a very low average molecular gas column density was measured. No significant local features were found that could hint of peculiar conditions in the ISM close to the GC.

\subsection{Far infrared data}

The Infrared Astronomical Satellite (IRAS) archival data, here from the IRIS data reduction chain (Miville-Deschêne \& Lagache 2005), provides full-sky coverage at 4 wavelengths (12, $25,60$ and $100 \mu \mathrm{m})$. The angular resolution $\left(4.2^{\prime}\right.$ at $\left.100 \mu \mathrm{m}\right)$ is sufficient to distinguish differences on the scale of the regions considered here. Bright point-like sources from the IRAS catalogue (Beichman et al. 1988) were removed when estimating the average flux in the regions. After comparison to Spitzer maps ${ }^{4}$, the limiting flux for source exclusion was set in the $12 \mu \mathrm{m}$ band, where the contribution from stars is largest, to exclude at least the two IRAS point-like sources ${ }^{5}$ closest to Terzan 5, corresponding to the GC itself and a nearby bright star.

A flux density gradient is observed across the region, with a factor 2 decrease when moving away from the Galactic plane, with the lowest fluxes measured for region 12, the furthest away from the Galactic plane. Visual inspection of IRAS and Spitzer maps did not reveal any obvious extended structure in this region apart from Terzan 5 itself, where star crowding would mask any truly extended emission component.

\subsection{Molecular gas and ${ }^{12} \mathrm{CO}$ data}

The largest-coverage molecular-gas-tracer map of the Galactic plane was compiled by Dame et al. (2001), hereafter DA01, from the data of several surveys of the $\mathrm{CO}$ rotational transition 1-0 emission line. We extracted the temperature values against velocity from their publicly available ${ }^{6}$ data cube. The values were integrated over the relevant velocity range, over the spatial extension of the region and finally converted into an hydrogen column density $n_{\mathrm{H}_{2}}\left(\mathrm{~cm}^{-2}\right)$ using the conversion factor $1.8 \times 10^{20} \mathrm{~cm}^{-2} \mathrm{~K}^{-1} \mathrm{~km}^{-1} \mathrm{~s}$ from DA01.

Using the close-case distance estimates from a Galactic rotation model (here following Clemens 1985; Nakanishi \& Yoshiabi 2003), we selected the boundary velocities to bracket the distance to Terzan $5(5.9 \mathrm{kpc}): 20 \mathrm{~km} \mathrm{~s}^{-1}(\approx 5 \mathrm{kpc})$ and $120 \mathrm{~km} \mathrm{~s}^{-1}(\approx 7.3 \mathrm{kpc})$. The complete range provided by DA01 was also considered (from minus to plus $170 \mathrm{~km} \mathrm{~s}^{-1}$ ). The estimated $n_{\mathrm{H}_{2}}$ are very low, as illustrated in Fig. 3, compatible with zero in some regions, notably region 12 in all velocity ranges. The maximum values are measured in regions 6 and 9, with respectively 2.6 and $4.5 \times 10^{21} \mathrm{~cm}^{-2}$ in the full velocity range and 3.4 and $3.3 \times 10^{20} \mathrm{~cm}^{-2}$ in the velocity range compatible with the distance to Terzan 5 . These values are comparable to

\footnotetext{
${ }^{4}$ http://irsa.ipac.caltech.edu/applications/Cutouts/

5 Catalogue accessed from Vizier (Ochsenbein et al. 2000).

${ }^{6}$ http://www.cfa.harvard.edu/mmw/obtainingData.html
} 


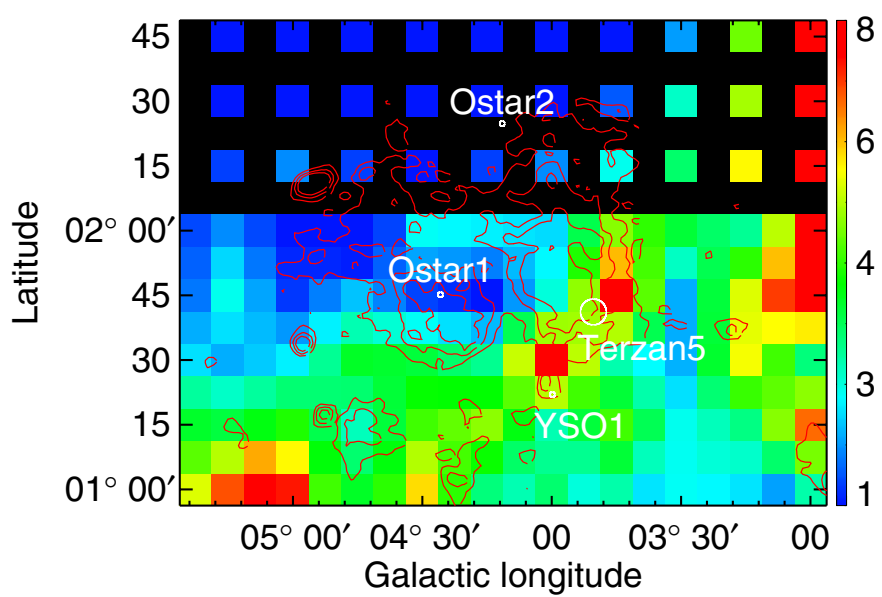

Fig. 3. Map of $n_{\mathrm{H}_{2}}$ derived from the ${ }^{12} \mathrm{CO}$ emission in the 3 to $10 \mathrm{~km} \mathrm{~s}^{-1}$ velocity range (roughly equivalent to a distance range of 1 to $3 \mathrm{kpc}$ ) overlaid with contours from the $11 \mathrm{~cm}$ data (for levels $0.07,0.095$ and $0.12 \mathrm{Jy} /$ beam) and selected objects (see Sect. 5). The Terzan 5 circle indicates the half-mass radius of the GC. Colour scale normalised by $10^{20} \mathrm{~cm}^{-2}$. The map was blanked where no value was available.

other regions at similar Galactic latitude, where the latitude gradient of $n_{\mathrm{H}_{2}}$ was shown to be large (DA01). The neighbouring giant molecular cloud Bania Clump 2 mentioned in DA01 does not extend to this region. Most of the measured column density is associated to velocity range $v<20 \mathrm{~km} \mathrm{~s}^{-1}$ (distances below $5 \mathrm{kpc}$ ). We interpret it as the cumulative emission from the diffuse molecular gas near the Galactic plane along the line of sight. Marginally significant structures found from visual inspection of the data cube are discussed in Sect. 5. The slightly larger values of $n_{\mathrm{H}_{2}}$ for regions 6 and 9 correspond to the shorter distance of these regions to the Galactic plane. Region 12 is the furthest away from the Galactic plane, away from distinct high-latitude features, so the null result (to the sensitivity of the available data) is not surprising.

\section{Catalogue search}

Previously identified objects potentially relevant to our study were searched with the help of the CDS online tools ${ }^{7}$. There appears to be no particularly energetic object in the region that could straightforwardly generate the entire observed radio emission. A few objects are worth mentioning (shown in Fig. 4). The large radio source east of region 12 is SNR WR143. Two active galactic nuclei have been identified, one on each side of region 12 , both as bright radio point sources. Two $\mathrm{O}$ type stars are found, one near the centre of region 10, the other north of Terzan 5. Both stars seem to be at a distance below $2 \mathrm{kpc}$ (Reed 2005). Figure 3 shows $n_{\mathrm{H}_{2}}$ for a compatible velocity range, with a local minimum coincident with one of the stars, labelled "Ostar1" in the figure and catalogued as 2MASS J174910162414211. This may hint of a previous interaction between the star and molecular gas leading to a cavity. The data is too sparse near the other star to make any statement. Based on the IRAS source catalogue (Beichman et al. 1988) and young stellar object (YSO) selection criteria from IRAS colours (Junkes et al. 1992), two candidate YSOs were identified, one of them (labelled as "YSO1") close to a radio hot spot and possibly a ${ }^{12} \mathrm{CO}$ one. All the other bright radio hot spots were previously catalogued

\footnotetext{
${ }^{7}$ http://cdsweb.u-strasbg.fr/
}

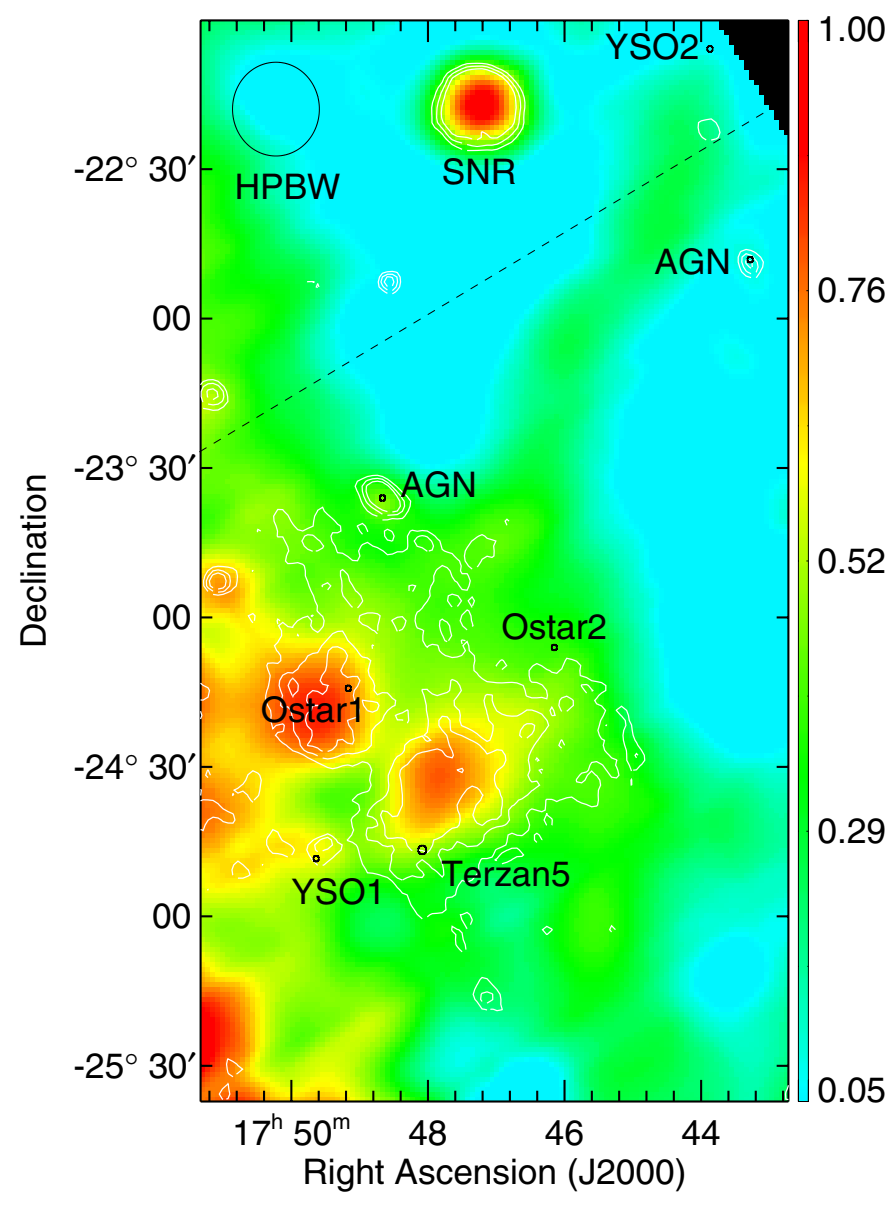

Fig. 4. Radio map from the Effelsberg Galactic plane surveys at $21 \mathrm{~cm}$ (in Jy/beam). Contours from the $11 \mathrm{~cm}$ map (for levels 0.07, 0.095 and $0.12 \mathrm{Jy} / \mathrm{beam})$, selected catalog objects as well as the $H P B W$ for the $21 \mathrm{~cm}$ data are over-plotted. The Terzan 5 circle indicates the half-mass radius of the GC. The dashed line indicate the radio scan direction at $21 \mathrm{~cm}$.

(Reich et al. 1990a) but we found no additional published information on them.

\section{Constraints on non-thermal emission processes}

Based on the collected measurements, this section focuses on testing non-thermal radiation processes that may generate the emission around Terzan 5. The energetics of the detected radio structures are constrained in Sect. 6.1. The particular properties of regions 10, 11 and 12 are discussed in Sects. 6.2 to 6.4. Potential associations between Terzan 5 and region 11 or 12 are discussed. Section 6.5 examines the origin of the diffuse X-ray emission found by Eger et al. (2010).

While a physical connection between the structures seen around the direction of Terzan 5 (Figs. 1 and 4) and the GC itself cannot be affirmed, it is assumed hereafter that they are all located at the same distance of $5.9 \mathrm{kpc}$. For the interpretation of the radio data, it is further assumed that synchrotron radiation (SR) emission is the dominant scenario. If the electron energy distribution follows a power law of index $p, N(E) \mathrm{d} E=\kappa E^{-p} \mathrm{~d} E$, the radio spectral index $\alpha$ of the SR emission is $\alpha=(p-1) / 2$. Typical values (Longair 1992) for $\alpha$ are: for centre-filled supernova remnants (SNR) $\alpha \approx-0.3-0.0$, for SNR shells $\alpha \lesssim-0.5$ and for the Galactic radio $\mathrm{GHz}$ range emission $\alpha \approx-0.9-0.8$. In the absence of radio index measurements, we assume for all 
Table 3. Magnetic field strength, energy density and energetics for the regions computed according to the MEC.

\begin{tabular}{lcccccccccccc}
\hline \hline & & \multicolumn{1}{c}{ Region } & \multicolumn{1}{c}{} \\
& 1 & 2 & 3 & 4 & 5 & 6 & 7 & 8 & 9 & 10 & 11 & 12 \\
\hline$B_{\mathrm{me}}[\mu \mathrm{G}]$ & 10.3 & 8.9 & 10.3 & 8.1 & 7.2 & 9.1 & 9.3 & 7.9 & 5.7 & 11.4 & 9.5 & 5.6 \\
$u_{\mathrm{me}}\left[10^{-12} \mathrm{erg} \mathrm{cm}^{-3}\right]$ & 9.8 & 7.4 & 9.8 & 6.1 & 4.8 & 7.7 & 8.0 & 5.8 & 3.0 & 12 & 8.4 & 2.9 \\
$E_{\mathrm{me}}\left[10^{48} \mathrm{erg}\right]$ & 2.1 & 8.1 & 11 & 6.7 & 5.3 & 19 & 20 & 15 & 7.5 & 2.8 & 12 & 29 \\
\hline
\end{tabular}

Notes. See text for details.

regions $\alpha=-0.8$. Along with this, a Bremsstrahlung scenario is investigated for region 10 .

\subsection{Synchrotron radiation energetics}

To estimate the energy in the radio structures assuming SR emission, the minimum energy condition (MEC) is used, as reviewed in Miley (1980). The MEC density of a radio source can be written as $u_{\mathrm{me}}=(7 / 3)\left(B_{\mathrm{me}}^{2} / 8 \pi\right) \mathrm{erg} \mathrm{cm}^{-3}$ and corresponds almost (but not exactly) to the equi-partition of energy between non-thermal particles and the magnetic field. For the frequency interval $v=0.01-100 \mathrm{GHz}$ and assuming a spectral index $\alpha=-0.8$, the corresponding magnetic field, in $\mu \mathrm{G}$ (for frequencies in $\mathrm{GHz}$ ), is given by:

$B_{\mathrm{me}}=140\left(\frac{v_{0}}{1 \mathrm{GHz}}\right)^{0.22}\left[\frac{S_{0}}{(1 \mathrm{Jy})} \frac{\left(1 \operatorname{arsec}^{2} 1 \mathrm{kpc}\right)}{\theta_{x} \theta_{y} s}\right]^{2 / 7}$

where $\theta_{x}$ and $\theta_{y}$ are the extent of the radio source on the plane of the sky in orthogonal directions, $s$ is the path length through the source along the line of sight and $v_{0}$ is the frequency where the flux $S_{0}$ is measured.

The MEC is assessed from the measurements of Table 2, for $v_{0}=2.7 \mathrm{GHz}$. It is assumed that region 1 is spherically symmetric with a radius of $15 \mathrm{pc}$ and that regions 3 to 10 extend by the same $30 \mathrm{pc}$ along the line of sight $(s=30 \mathrm{pc})$. The regions 10 to 12 are assumed to be structures where $s$ equals the minimum extension of the source in the plane of the sky, estimated here for a distance of $5.9 \mathrm{kpc}$. Since the strength of $B_{\text {me }}$ scales only with $\left(S_{0} / s\right)^{2 / 7}$, the uncertainties on the actual path lengths and the flux measurements are not critical to derive orders of magnitude. Under these assumptions, energetics for all the regions fall between a few $10^{48} \mathrm{erg}$ and more than $10^{49} \mathrm{erg}$. The values for $u_{\mathrm{me}}, B_{\mathrm{me}}$ as well as the total energy $E_{\mathrm{me}}$ (in particles and in the magnetic field) in each region are given in Table 3 . These values serve as references in the following region-by-region discussions.

\subsection{The $O$ star and region 10}

Region 10 is located north-east of Terzan 5. It does not overlap with the GC but contains an $\mathrm{O}$ star at a distance shorter than $2 \mathrm{kpc}$ (see Sect. 5). Even though an association with the GC cannot be excluded, we now assume that region 10 is related to the $\mathrm{O}$ star, which could serve as source of ionising radiation for a Bremsstrahlung scenario. There are indications for a hole in the ${ }^{12} \mathrm{CO}$ distribution at distances between 1 and $3 \mathrm{kpc}$ consistent with the location and potentially the distance of the $\mathrm{O}$ star. In the Bremsstrahlung picture the $\mathrm{O}$ star would ionise the embedding molecular material causing this hole in the ${ }^{12} \mathrm{CO}$ distribution. The ionised material would then emit Bremsstrahlung (e.g. Vasquez et al. 2010). In such a scenario, $n_{\mathrm{e}}$ would be comparable to the molecular gas density, of the order of $1-10 \mathrm{~cm}^{-3}$. A flux density from region 10 of $14\left(T / 10^{4} \mathrm{~K}\right)\left(n_{\mathrm{e}} / 10 \mathrm{~cm}^{-3}\right) \mathrm{Jy}$ would be expected, in broad agreement with the observed value. The expected Bremsstrahlung spectrum would be flat in the radio range ( $\alpha \approx-0.1$ ). As visible in Fig. 4 , the features at 11 and $21 \mathrm{~cm}$ corresponding to region 10 do not match exactly. The possibility remains therefore that the set of properties used here for this region do not accurately describe it. Observation at higher spatial resolution may settle this issue.

\subsection{Accumulation of relativistic electrons in region 11}

The enhanced emission in region 11 covers the location of Terzan 5 and extends from there to the north-west of the GC, away from the Galactic Plane.

\subsubsection{Evidence from the radio maps}

The radio emission in region 11 represents in the MEC a total energy of $9.2 \times 10^{48} \mathrm{erg}$. For SR emission peaking at a few $\mathrm{GHz}$ in the MEC magnetic field of about $10 \mu \mathrm{G}$ (see Table 3), electrons with an energy of about $10 \mathrm{GeV}$ would be required (see e.g. Longair 1992). Following Aharonian (2004), the cooling time of these electrons in such a magnetic field would be about $10^{7}$ years. This scenario could be tested by the radio index, as radio spectral indices of $-0.3-0.0$ are typical of pulsar wind nebulae (PWNs, see Gaensler \& Slane 2006). Sampling the radio index within region 11 could then be used to estimate the electron production time scale, expected to be shorter than the cooling time if no spectral steepening was found.

\subsubsection{Possible association with Terzan 5}

Given the geometry of the radio feature covered by region 11 , the large population of msPSRs in the GC could be the source of the radio-emitting electrons. Assuming that Terzan 5 contains 180 msPSRs (Abdo et al. 2010), each injecting energy on average at a moderate rate of $10^{33} \mathrm{erg} / \mathrm{s}$ in electrons into the surroundings, about $2 \times 10^{6}$ years would be needed to build up the total electron population. This time scale is shorter than the cooling time estimated above, so this scenario seems viable.

The radio structure covered by region 11 extends from Terzan 5 in a direction roughly perpendicular to the Galactic plane. Based on the energy density given in Table 3 and assuming for the ISM a density of $0.1 \mathrm{~cm}^{-3}$ and a temperature of $10^{5} \mathrm{~K}$ (see e.g. Aavage \& de Boer 1981), this radio structure would seem to be mildly over-pressured (by a factor of 3 ) with respect to the surrounding gas. It could therefore be expected to expand, preferentially in the direction of the Galactic density gradient, as observed. In this scenario, the expansion of the bubble is driven by the difference between the internal and external pressures ( $p_{\text {int }}$ and $p_{\text {ext }}$ ) over the surface $S$ where they exert, with $F_{\mathrm{p}} \sim\left(p_{\text {int }}-p_{\text {ext }}\right) \times S$. It is limited by the ram pressure of the surrounding medium, $F_{\text {ram }} \sim C \frac{1}{2} S v^{2} \rho_{\text {ext }}$ according to Churazov et al. (2001), with $C$ the drag coefficient, $v$ the expansion velocity 
and $\rho_{\text {ext }}$ the density outside the bubble. The maximum terminal expansion velocity is then given by:

$v \sim \sqrt{\frac{2}{C} \frac{p_{\mathrm{int}}-p_{\mathrm{ext}}}{\rho_{\mathrm{ext}}} .}$

For $C=0.75$ (Churazov et al. 2001), $v \sim 80\left(n / 0.1 \mathrm{~cm}^{-3}\right) \mathrm{km} \mathrm{s}^{-1}$. At this constant velocity, of the order of $10^{6}$ years are needed to expand by $100 \mathrm{pc}$. These numbers are in rough agreement with the production timescale of the electron population in region 11.

Another mechanism operating at the location of Terzan 5 could potentially displace radio-emitting plasma produced in the $\mathrm{GC}$ in the direction perpendicular to the Galactic plane. Terzan 5 is placed in the onset region of the Galactic wind of our Galaxy, where the bulk motion flow of the wind material is expected to exceed $100 \mathrm{~km} \mathrm{~s}^{-1}$ (Everett et al. 2008). This Galactic wind could push away material originating in Terzan 5 even faster than the pressure imbalance discussed in the previous paragraph.

Detection of radio polarisation in this region, challenging at the distance of Terzan 5 (see e.g. the discussion on polarisation horizon in Landecker et al. 2010), would support a plasma plume scenario. These dynamical models could be further tested against the proper motion of the GC. For instance, in $2 \times 10^{6}$ years and for a velocity in the plane of the sky of $50 \mathrm{~km} \mathrm{~s}^{-1}$, a $100 \mathrm{pc}$ long structure could be generated in the direction of motion. Conversely, too high a velocity or an incompatible direction of proper motion would exclude the proposed association between the radio feature in region 11 and Terzan 5.

\subsection{The large-scale ridge in region 12}

The ridge-like radio structure seen in region 12 stands out for its length and apparent alignment with Terzan 5. It is more visible in the $21 \mathrm{~cm}$ map (see Fig. 4). Structures extending over similar linear scales and involving comparable energetics have been proposed (Fukui et al. 2009; Yamamoto et al. 2008) to result from highly collimated outbursts from either micro-quasars or stellar explosions. Such objects may be hosted by GCs like Terzan 5 but no equivalent central object or active region could be identified in relation to region 12 . Higher sensitivity observations and dedicated investigations appear to be necessary to establish a full emission scenario for this region.

\subsection{Revisiting the diffuse $X$-ray emission around Terzan 5}

\subsubsection{Non-thermal Bremsstrahlung}

The molecular gas and infrared data can be used to test a nonthermal Bremsstrahlung origin for the extended diffuse X-ray emission in Terzan 5. In this scenario, electrons are deflected by target nuclei from the ambient medium. Therefore the intensity of the non-thermal X-ray emission should be correlated with the density of the local ISM. The absence of observational support from the ${ }^{12} \mathrm{CO}$ or infrared data for regions with high density of target material goes toward excluding a nonthermal Bremsstrahlung scenario for the diffuse X-ray emission in Terzan 5. More detailed investigation of the infrared data as well as higher resolution molecular gas tracer data may change this conclusion.

\subsubsection{Inverse Compton scenario}

One possible origin for the diffuse X-ray emission discovered by Eger et al. (2010) is X-ray IC radiation, usually accompanied by radio SR emission. The data gathered in this work can constrain this scenario.

The predicted radio SR emission is described by the frequency $v_{\text {syn }}=120\left(\gamma / 10^{4}\right)^{2}(B / 1 \mu \mathrm{G}) \sin \phi \mathrm{MHz}$, with $\gamma$ the Lorentz factor of the electrons, $B$ the magnetic field and $\sin \phi$ the pitch angle of the electrons with respect to the magnetic field. For $\gamma \approx 30$, typical of IC radiation in the $\mathrm{keV}$ range (see Krockenberger \& Grindlay 1995), and neglecting $\sin \phi$, one gets $v_{\text {syn }}=1.2(B / 1 \mu \mathrm{G}) \mathrm{kHz}$. The SR flux relates to the IC flux by $F_{\text {syn }} / F_{\text {IC }} \approx\left(B^{2} / 8 \pi\right) / u_{\text {rad }}$, where $B^{2} / 8 \pi$ is the magnetic field energy density and $u_{\text {rad }}$ is the radiation energy density responsible for the IC up-scattering. Assuming 1) that the X-ray flux of $5.5 \times 10^{-13} \mathrm{erg} \mathrm{cm}^{-2} \mathrm{~s}^{-1}$ found by Eger et al. (2010) originates from IC emission; 2) that the MEC applies, giving a magnetic field $B=10.3 \mu \mathrm{G} ; 3)$ that the radiation field is $u_{\mathrm{rad}}=40 \mathrm{eV} / \mathrm{cm}^{3}$ (Eger et al. 2010), a flux density of the order of $300 \mathrm{kJy}$ at the related synchrotron frequency of about $12 \mathrm{kHz}$ is expected. Extending a power-law spectrum up to the $\mathrm{GHz}$ range would require a spectral index around -1.0 for region 1 .

The apparent validity of the IC emission hypothesis for the diffuse X-ray emission found by Eger et al. (2010) could be further tested with an accurate radio spectral index.

\section{Conclusion}

In this paper the surroundings of the GC Terzan 5 have been searched for indications of non-thermal diffuse emission. Several structures were isolated in the Effelsberg 21 and $11 \mathrm{~cm}$ surveys. The currently available data point towards a complex radio emitting region. However, interpretation of the radio structures is limited in the absence of reliable radio index. Future observations with high-frequency single-dish radio-telescopes or a dedicated re-analysis of the original Effelsberg data would improve this situation.

The most intriguing of these structures extends from the position of the GC to about $0.8^{\circ}$ to the north-west. The energetics of this structure could be produced on a reasonable time scale by the population of msPSRs in Terzan 5 in a PWN scenario. Comparison of these time scales with the GC proper motion would provide a strong test to this model.

A smaller compact radio feature may be associated with an $\mathrm{O}$ star. Indication for a molecular gas cavity and order of magnitude agreement between the radio flux and a Bremsstrahlung model support this scenario. Confirmation of the molecular gas feature and of its distance are necessary tests to the proposed association.

There is an intriguing ridge-like extended radio source aligned with Terzan 5 roughly $1^{\circ}$ north-east of the GC. In terms of energy content and spatial extent it shows similar characteristics to proposed remnants of highly asymmetric outbursts, either from a micro-quasar or the final explosion of a star. Since a GC may be the host of highly asymmetric explosions, such an origin cannot be excluded.

The presented multi-wavelength data is compatible with an IC origin for the recently discovered extended diffuse X-ray emission from the direction of Terzan 5. A non-thermal Bremsstrahlung scenario is not supported, although higher resolution data may change this conclusion.

Likewise, several negative results were obtained. No significant density of molecular material in the environment of Terzan 5 could be found, nor any clear hint of peculiar dust emission structure in the (low resolution) infrared data. The extension of the diffuse X-ray emission was limited to a 2.5 radius region around Terzan 5, to the sensitivity limit of the data. 
Acknowledgements. The authors thank Karl-Heinz Mack (INAF, Istituto di Radioastronomia Bologna, Italy) for discussions about the radio analysis and Matthieu Renaud (CNRS, LPTA Montpellier, France) for sharing his expertise on INTEGRAL. This work was supported in part by the Polish Ministry of Science and Higher Education project N N203 380336.

\section{References}

Abdo, A. A., Ackermann, M., Ajello, M., et al. (The Fermi/LAT, collaboration), 2009, Science, 325, 845A

Abdo, A. A., Ackermann, M., Ajello, M., et al. (The Fermi/LAT, collaboration), 2010, A\&A, 524, 75

Aharonian, F. A. 2004, Very high energy cosmic gamma radiation: a crucial window on the extreme Universe (World Scientific Publishing)

Baars, J. W. M., Genzel, R., Pauliny-Toth, I. I. K., et al. 1977, A\&A, 61, 99

Bednarek, W., \& Sitarek, J. 2007, MNRAS, 377, 920

Beichman, C., et al. 1988, IRAS Catalogs and Atlases Explanatory Supplement, NASA RP-1190, 1

Blackburn, J. K. 1995, ASP Conf. Ser., 77

Boese, F. G. 2000, A\&AS, 141, 507B

Churazov, E., Brüggen, M., Kaiser, C. R., et al. 2001, ApJ, 554, 261

Clemens, D. P. 1985, ApJ, 295, 422C

Condon, J. J., Cotton, W. D., Greisen, E. W., et al. 1998, AJ, 115, 1693

Dame, T. M., Hartmann, D., \& Thaddeus, P. 2001, ApJ, 547, 792D

David, L. P., et al. 1997, The ROSAT High Resolution Imager calibration report, Harvard-Smithsonian Center for Astrophysics, ftp://legacy.gsfc . nasa. gov/rosat/doc/hri/hri_report

Domainko, W., \& Ruffert, M. 2005 A\&A, 444, L33

Domainko, W., \& Ruffert, M. 2008, AdSpR, 41, 518

Draine, B. T., \& Li, A. 2007, ApJ, 657, 810D

Eger, P., Domainko, W., \& Clapson, A.-C. 2010, A\&A, 513, 66

Everett, J. E., Zweibel, E. G., Benjamin, R. A., et al. 2008, ApJ, 674, 258

Fender, R. P., Garrington, S. T., McKay, D. J., et al. 1999, MNRAS, 304, 865

Ferraro, F. R., Beccari, G., Dalessandro, E., et al. 2009, Nature, 462, 1028

Fruchter, A. S., \& Goss, W. M. 2000, ApJ, 536, 865

Fukui, Y., Furukawa, N., Dame, T. M., et al. 2009, PASJ, 61, L23

Gaensler, B. M., \& Slane, P. O. 2006, ARA\&A, 44, 17

Gopal-Krishna, \& Steppe, H. 1980, A\&A, 88, 354

Green, D. A. 2009, BASI, 37, 45

Grindlay, J., Portegies, Z. S., \& McMillan, S. 2006, Nature Phys., 2, 116

Harris, W. E. 1996, AJ, 112, 1487
Heinke, C. O., Grindlay, J. E., Edmonds, P. D., et al. 2002, BAAS, 34, 1313

Heinke, C. O., Wijnands, R., Cohn, H. N., et al. 2006, ApJ, 651, 1098

Isobe, N., Tashiro, M., Makishima, K., et al. 2002, ApJ, 580, L111

Ivanova, N., Heinke, C. O., Rasio, F. A., Belczynski, K., \& Fregeau, J. M. 2008, MNRAS, 386, 553

Johnston, H. M., Verbunt, F., \& Hasinger, G. 1995, A\&A, 298, L21

Junkes, N., Fuerst, E., \& Reich, W. 1992, A\&A, 261, 289

Karovska, M., Beikman, S. J., Elvis, M. S., et al. 2001, ASP Conf. Proc., 238, 435

Klein, U., Mack, K.-H., Gregorini, L., \& Vigotti, M. 2003, A\&A, 406, 579

Kong, A. K. H., Hui, C. Y., \& Cheng, K. S. 2010, ApJ, 712, 36

Koyama, K., Petre, R., Gotthelf, E. V., et al. 1995, Nature, 378, 255

Krivonos, R., Revnivtsev, M., Tsygankov, S., et al. 2010, A\&A, 519, 107

Krockenberger, M., \& Grindlay, J. E. 1995, ApJ, 451, 200K

Landecker, T. L., Reich, W., Reid, R. I., et al. 2010, A\&A, 520, 80

Lanzoni, B., Ferraro, F. R., Dalessandro, E., et al. 2010, ApJ, 717, 653

Lesch, H., \& Reich, W. 1992, A\&A, 264, 493

Longair, M. S. 1992, High energy astrophysics, 1: Particles, photons and their detection (Cambridge University Press)

Miley, G. 1980, ARA\&A, 18, 165

Miville-Deschênes, M.-A., \& Lagache, G. 2005, ApJS, 157, 302

Nakanishi, H., \& Yoshiaki, S. 2003, PASJ, 55, 191N

O’Dea, C. P., Daly, R. A., Kharb, P., et al. 2009, A\&A, 494, 471

Ochsenbein, F., Bauer, P., \& Marcout, J. 2000, A\&AS, 143, 221

Okada, Y., Kokubun, M., Yuasa, T., \& Makishima, K. 2007, PASJ, 59, 727

Paladini, R., Burigana, C., Davies, R. D., et al. 2003, A\&A, 397, 213

Perner, R., Raymons, J., \& Loeb, A. 2000, ApJ, 533, 658

Pooley, D., \& Hut, P. 2006, ApJ, 646, L143

Ransom, S. M. 2008, in IAU Symp. 246, ed. E. Vesperini, M. Giersz, \& A. Sills, 291

Reed, B. C. 2005, AJ, 130,1652

Reich, W., Wielebinski, R., Sofue, Y., et al. 1988, A\&A, 191, 303

Reich, W., Reich, P., \& Fürst, E. 1990a, A\&AS, 83, 539R

Reich, W., Fürst, E., Reich, P., \& Reif, K. 1990b, A\&AS, 85, 633

Rybicki, G. B., \& Lightman, A. P. 1979, Radiative processes in astrophysics (New York: Wiley-Interscience)

Savage, B. D., \& de Boer, K. S. 1981, ApJ, 243, 460S

Shara, M. M., \& Hurley, J. R. 2002, ApJ, 571, 716

Vasquez, J., Cappa, C. E., Pineault, S., \& Duronea, N. U. 2010, MNRAS, 405, 1976

Venter, C., \& de Jager, O. C. 2008, AIPC, 1085, 277

Venter, C., de Jager, O. C., \& Clapson, A.-C. 2009, ApJ, 696, L52

Yamamoto, H., Ito, S., Ishigami, S., et al. 2008, PASJ, 60, 715 